\title{
0 mandato de administração das antigas colônias dos paises vencidos na guerra de 1914-1918
}

\author{
Prof. Vicente Ráo
}

Finda a guerra de 1914-1918, o território do sudoeste africano, que antes pertencera à Alemanha, foi entregue à União Sul-Americana, por intermédio de Sua Majestade Britânica, para ser administrado em nome da Sociedade das Nações.

Extinta a Sociedade das Nações, entendeu a União Sul-Africana de se esquivar do "contrôle" administrativo da Onganização das Nações Unidas, sob o fundamento de que, com o desaparecimento do mandatário desapareceu também o mandato.

A Côrte Internacional de Justiça, consultada, opinou em sentido contrário a essa pretensão; mas a União SulAfricana não se conformou com êste parecer e a questão foi de novo suscitada, perante a ONU.

Em nome da delegação brasileira, o Prof. Vicente Ráo pronunciou, então, o seguinte discurso, cujas conclusões foram adotadas pelas demais delegações:

"Pour nous prononcer sur la question du Sud-Ouest Africam, il faut, d'abord, procéder à une mise au point des termes du problème, en nous rapportant à la sourcs la plus objective possible, c'est'à-dire, à l'exposé qui précède l'Avis de la Cour Internationaie de Justice.

1. La destinée des anciennes colonies des pays qui ont perdu la guerre de 1914- 1918 a été décidé selon les 
deux principes suivants, retenus comme d'importance capitale: celui de la non-annexion à aucun pays déter. miné et celui selon lequel le bien-être et le développement des peuples de ces colonies seraient considérés comme "une mission sacrée de civilisation".

2. En obéissant exactement à ces principes, le régime international des Mandats fût créé par l'art. 22 du Pacte de la Société des Nations et à ce propos l'avis de la Cour Internationale de Justice remémore:". . une tutelle devrait être constituée pour ces peuples et cette tutelle devait être confiée à certaines nations développées et exercée par elles en qualité de Mandataires et au nom de la Société des Nations"

3. C'est ainsi que le territoire du Sud-Ouest Africain est passé du régime antérieur de soumission à la souveraineté de l'Allemagne, au régime nouveau de la soumission à une tutelle internationale exercée par la Société des Nations représentée par une Puissance Administrante.

4. Cette délégation de pouvoirs a reçu, alors, le nom de "Mandat".

Signalons tout de suit cet élément de très large portée, une vraie prémisse qui d'or et déjà se pose pour la solution logique et juridique du problème: - le caractère international de la tutelle et, conséquemment, du Mandat.

5. Les Principales Puissances Alliées et Associées ont, ainsi, attribué le Mandat d'Administration du territoire du Sud-Ouest Africain à Sa Majesté Britannique, pour être exercé en son nom par l'Union de l'Afrique du Sul. La Société des Nations, à son tour, a confirmé cette délégation de pouvoirs et en a défini les termes. 
6. Voici les clauses principales du Mandat: a) d'un coté, l'Union Sud-Africaine recevait pleins pouvoirs d'administration sur le territoire du Soud-Ouest Africain qui, à cet effet, serait considéré "comme partie intégrante de l'Union". Le Mandataire était, en conséquence, autorisé à appliquer au territoire sous Mandat les lois de l'Union, sous réserve des modifications nécessaires aux conditions locales; b) - de l'autre coté, l'Union de l'Afrique du Sud assumait, selon l'Avis de la Cour Internationale de Justice "des obligations internationales de deux sortes: - "les unes concernaient directement l'Administration du territoire et correspondaient à la "mission sacrée de civilisation" mentionnée à l'art. 22 du Pacte; les autres avaient trait au mécanisme de mise en oeuvre et étaient étroitement liées à la surveillance et aux fonctions de contrôle de la Société des Nations. Elles correspondaient aux "garantie pour l'accomplissement de cette mission", mentionnée dans le même article"

7. Les obligations les plus remarquables de ce prepremier groupe (art. 22 du Pacte et 2 à 5 du Mandat) se rapportent à la propulsion du bien-être matériel et moral et du progrès des habitants du territoire; à la liberté de conscience et au libre exercice du culte, y comprises des obligations spéciales à l'égard des missionaires; à la traite d'esclaves, au travail forcé, au trafic d'armements et de munitions et aux établissements militaires.

La nature de ces obligations dicte, à son tour, le caractère de fonction internationale de l'Administration confiée au Gouvernement de l'Afrique du Sud.

8. D'après les termes du Mandat, le territoire du Sud-Ouest Africain serait administré "comme une partie intégrante" de l'Union. Mais on ne doit attribuer à cette expression aucune portée spéciale, étant donné qu'elle ne 
visait d'autre finalité que de rendre l'Administration plus. facile.

Dans les Unions Administratives permises par les $\mathrm{Na}$ tions Unies, cette expression est aussi employée et, à ce propos, on peut lire dans le dernier Rapport du Conseil de Tutelle (texte français, pg. 206) l'explication suivante,

“. les représentants de la Belgique et de la France ont affirmé que "tels que les entendaient leurs gouvernements, les mots "comme partie intégrante” étaint nécessaires pour faciliter l'Administration et ne signifiaient pas que les gouvernements de la France et de la Belgique fussent autorisés à réduire l'individualité politique des ter-ritoires sous tutelle. Le Représentant du Royaume Uni a déclaré que les mots "comme partie intégrante" n'impliquaient pas que les territoires seraient administrés comme parties intégrantes du Royaume Uni proprement dit, ni que la souveraineté britannique s'étendrait à ces régions".

C'est à bonne raison, donc, que la Cour Internationale de Justiçe a affirmé: "il ressort des termes de ce Mandat ainsi que des dispositions de l'art. 22 du Pacte et des principes qui y sont énoncés, que la création de cette nauvelle institution internationale n'impliquait $n i$ cession de territoire, ni transfert de souverainetś à l'Union Sud Africaine. Le Gouvernement de l'Union devait exercer une fonction d'administration internationale au nom de la Société des Nations, aux fins de favoriser le bien-être et le développement des habitants".

Voilà comment la Cour vient de confirmer et de préciser la premisse que nous avons déjà signalée: 一 
c'est que nous nous nous trouvons vraiment em face d'une fonction d'administration internationale, exercée, en vertu de l'institution internationale de tutelle, par l'Union Sud Africaine, au nom de la Société des Nations.

9. L'Union conteste la survivance du Mandat, parce que la Société des Nations a cessé d'éxister; telle est la cause du différend qui a provoqué la consultation à la Cour Internationale de Justice (Résolutions de l'Assemblée Générale des Nations Unies, du 6septembre 1946, 141-II du ler. novembre 1947 et 227-III du 26 novembre 1948).

10. C'est vrai que, selon le droit commun interne de la généralité des Nations, quand le mandat cesse d'exister, le mandat lui-même perd son efficace. Mais cette perte n'est pas tranchante, ni absolue, car ce même droit commun reconnaît la nécessité et, conséquemment, la validité des actes pratiqués par le mandataire après l'extinction du mandat, quand ces actes sont indispensables pour la conservation et pour le maintien en valeur des biens ou des droits confiés à sa garde ou à son administration.

Le Mandaire reste toujours obligé, en ce cas, aux termes du mandat, malgré son extinction, et, en plưs, à la responsabilité légale qui se rattache à la gestion des biens ou des droits d'autrui.

Or, le territoire du Sud-Oust Africain n'appartenait et n'appartient pas à l'Union Sud Africaine et si nous acceptons la thèse, qu'elle soutient, de l'application du droit commun, nous aboutissons à la conclusion suivante: la fin de la Société des Nations n'importe pas la fin des obligations assumées par le mandataire, au moins jusqu'au moment de la création d'une nouvelle situation juridique résultante de la constitution d'un successeur du mandant extinct, c'est-à-dire, d'un successeur légalement habilité à 
contracter soit l'altération des powvoirs délégués, soit la cessation pure et simple de tous les affets du mandat.

Or, cette nouvelle situation juridique a été créée; ce sucesseur existe. Et nous examinerons, de suite, quelles sont les conséquences, sur les Mandats de ce genre, de cette nouvelle situation juridique et quelle est la portée des pouvoirs de ce successeur.

11. Mais la vraie solution juridique est tout autre, une fois que les règles qui disciplinent le mandat du. droit commun ne sont pas applicables au Mandat passé à l'Union de l'Afrique du Sud pour l'Administration du territoire du Sud-Ouest Africain.

La Cour Internationale de Justice s'est bien prononcée sur l'espèce, quand elle a dit:

"cette thèse (la thèse du gouvernement Soud Africain) est fondée sur une conception erronée $d \Im$ la situation juridique créée par l'arte. 22 dı Pacte et par le Mandat lui-même. La Société des Nations n'était pas, comme l'a allegué ce Gouvernement, un "mandant" au sens où ce terme est employé dans la législation de certains Etats. Elle avait seulemente assumé une fonction internationale de surveillance et de contrôle. Le Mandat n'avait de commun que le nom avec les notions, d'ailleurs diverses, du mandat en droit interne. Le but du Mandat régi par les: règles internationales dépassait de beaucoup celui des rapports contractuels régis par un droit national".

II n'y a d'assimilation possible, donc, entre le mandat du droit commun et le Mandat du droit international qui a pour objet. l'administration du Sud Ouest Africain. 
S'il en est ainsi, il faut bien se demander, tout-demême, quelle est la nature juridique du "Mandat" conféré par la Société des Nations à Sa Majesté Britannique, pour être exercé par le Gouvernement de l'Afrique du Sud.

La réponse ne sera dificile, attendu que la Cour Internationale de Justice n'a pas renoncé du tout à la recherche de cette qualification juridique. Bien au contraire, à ce propos, elle nous enseigne et renseigne:

"que le Mandat (pour les anciennes colonies) a été créé dans l'intérêt des habitants des territoires et de l'Humanité en général, comme une mission internationale là laquelle était assignée un but international: - une mission sacrée de civilisation - Les règles internationales régissant le Mandat constituaient pour le territoire un statut international reconnu par tous les Membres de Ia Société des Nations, y compris l'Union Sud Africaine".

Ce Mandat ou peut le définir, donc, comme une institution internationale, qui a crée un statut international en bénéfice du Sud-Ouest fricain; et ce statut, une fois créé et reconnu par la communauté des Nations, ne peut pas être supprimé par l'acte unilatéral de l'Etat chargè simplement de l'Administration.

Nous avons, donc, suivi le bon chemin, en soulignant comme une prémisse le caractère international de la tutelle et, conséquemment, du Mandat et des fonctions mème attribuées au Mandataire.

Ainsi, l'invocation du droit commun est à rejeter: (a) en conséquence de la nature même du Mandat passé ail Gouvernement Sud-Africain et (b) parce qu'il serait absurde de pretendre (la Cour l'a bien reconnu elle aussi) que la fin du mandat fait périr les obligations du mandataire, tout en conservant les droits que de ce même mandat décou- 
lent. La règle jus et obligatio sunt correlata a cessé, dès longtemps, d'être une régle romaine, pour se transformer en règle universelle de droit.

12. C'est toujours vrai, tout-de-même, que les principes de droit, par leur universalité, régissent aussi bien dans le droit public national ou international, que dans droit privé de chaque peuple. Mais on ne doit pas confondre les principes généraux de droit, avec les règles particulières aux actes ou institutions júridiques en espèce, ainsi, par exemple, le mandat en droit privé, qu'on ne peut transporter, tel quel, ni dans le droit international, ni dans droit interne constitutionnel ou administratif.

Or, le principe général de droit, duquel le mandat n'est qu'une application spécifique, est, en ce qui nous intérèsse, celui de la représentation. Ce principe on peut l'énoncer d'une façon très générale, en disant qu'il y a une représentation toutes les fois que, par force de la loi ou de la convention, un acte juridique est pratiqué an nom et au profit d'autrui.

13. Ce concept de représentation est à la base d'une très grande quantité d'institutions juridiques, de droit privé ou de droit public interne ou externe, institutions qui sont aussi soumises ì des règles propres et particulières.

C'est ainsi que, par exemple, il y a une idée juridique de représentation, par force de la loi ou du contrat, a la base: - des droits paternels, de la tutelle et de la curatelle en droit de famille, pour suppléer l'incapacité des mineurs ou des personnes déclarées incapables; à la base du mandat stipulé par voie contractuelle ou simplement accepté; ì la base de toutes les affaires fiduciaires; de la médiation civile, du courlage et de toutes les formes de représentation commerciale; dans les substitutions par donations ou testaments; dans les associations civiles et les societés commerciales, quoique les partisans de la repré- 
sentation se disputent encore avec les partisans de la théorie organiciste; dans l'administration des patrimoine en succession ou en liquidation ou sous faillite; dans l'action du Ministère Public ou des Procureurs de l'Etat; dans les concessions ou autorisations pour l'exécution des services publiqués; das l'organisation de l'Etat, pour le choix des membres des pouvoirs politiques et, enfin, en droit international, pour nous détenir rien que sur le sujet en cause, constatons qu'il y a aussi une idée juridique de représentation à la base de la délegation de pouvoirs (sous le nom de Mandat) à un Etat déterminé, prour l'administration d'un territoire non-autonome. exercée au profit de la population de ce territoire et au nom de la Société des Nations.

Le trait commun à tous ces cas consiste en ce que Ze représentant agit toujours au nom et au profit d' autrui. Le mandat au profit du mandaire n'est qu'une forme exceptionnelle de mandat, qui déguise un transfert de droits et qui n'a, pour nous, ici, aucun intérêt, -aucune portée sur la matière en débat.

Bref, on peut parler, en l'espèce, de représentatioù au sens général du mot; mais on ne pourrait jamais invoquer les règles spécifiques, d'ailleurs si variables de législation à législation, qui disciplinent le mandat du droit commun.

14. Entre les institutions de droit commun et les institutions de droit public interne ou externe, des simiMitudes existent sans doute, quoicque elles soient plus formelles que de substance.

Considérée de ce point de vue, la représentation en droit international, dont nous nous ocupons, est plus proche des actes juridiques fiduciaires, que du mandat, en droit privé.

Nous voulons alluder aux actes fiduciaires au sens anglo-américain de "trust" et non au sens du droit latin, 
droit dans lequel de ces actes, les uns reçoivent une structure spécifique tout-à-fait diverse du droit du type anslais, et les autres présupposent une simulation à peine tolerée par la loi, - tandis que le "trust", sous toutes. ses lormes, est toujours protégé, en Angleterre et en Amérique, tout au moins par le droit - équité ("equity").

Sous un aspect purement analogique, on peut, donc, souscrire l'avis personnel, proféré en ce sens, prar l'éminent juge de la Cour Internationale de Justice, Sir Arnold McNair.

15. Mais tout en acceptant, par analogie, la qualification de "trust" donnée à ce Mandat par Sir Arnold McNair, nous ne pouvons, toutefois, concorder avec cet éminent juriste, quand il soutient:

“les obligations contractées envers la Société (des Nations) elle-même, sont éteintes. Les obligations contractẻes envers les anciens membres de la Société à la date où celle-ci a été dissoute, subsistent, sauf dans la mesure où leur exécution implique la coopération efective de la Société des Nations, ce qui est maintenant impossible. En outre, le statut international créé par le Soud-Ouest Africain, à savoir, celui d'un territoire gouverné par en Etat en vertu d'un titre limité, tel que ce titre est défini dans un Mandat, subsiste. Bien qu'il n'existe plus de Société des Nations pour serveiller l'exercice de ce Mandat, ce serait une erreur de croire que le mandataire n'est soumis à aucun contrôle. Tous les Etats qui faisaient partie de la Société des Nations à l'époque de sa dissolution, ont encore un intérét juridique à ce que le Mandat soit exercé comme il convient. Le mandat prévoit deux sortes de mécanismes: - un mécanisme judiciaire, résul- 
tant du droit que l'art. 7 réserve à tout le Membre de la Société des Nations de citer obligatoirement le Mandataire devant la Cour Permanente, - et un mécanisme administratif, com-prenant des rapports annuels et l'examen de ces derniers par la Commission Permanente des Mandats de la Société des Nations".

Selon l'auteur de cet avis personnel, la stirveillance judiciaire a été conservée expressement par l'art. 37 du Statut de la Cour Internationale de Justice, - ce qui est tout-à-fait vrai; mais, en revanche, la serveillance administrative a cessé d'exister, parce que la Société des Nations, son Conseil et la Commission Permanente des Mandats n'existent plus. II ne reste, donc, comme contrôle de l'exercice du Mandat, que le pouvoir des ancies Etats Membres de la Société de provoquer, au cas échéant, la décision de la Cour Internationale de Justice.

Or, c'est exactement la négation du contrôle administratif que nous contestons, fondés, d'ailleurs, sur des solides raisons, y comprises celles adoptées par la Cour.

16. Nous avons insistemment signalé, au cours de cette intervention, le caractère international de l'institution de tutelle, du Mandat et des fonctions délégués a l'Etat chargé de l'dministration, caractère qui n'est qu'une conséquente de la finalité envisagée par la Sociétè des Nations, - c'est-à-dire, l'accomplissement d'une "mission sacrée de civilisation", en bénéfice de l'humanité.

Et quand on parle d'institution internationale ayant cette finalité, ce serait réduire ce concept à très peu de chose que de limiter l'internationalité rien qu'aux rapports entre la Puissance Administrante et les anciens Etats-membres de la Société des Nations, considérés individuellement. 
Aucun traité ne fût jamais stipulé, d'ailleurs, en ce sens, entre ces Etats et l'Union Sud-fricaine. Dans l'acte conventionnel du Mandat, les parties étaient, d'un coté, la Société des Nations, douée elle-mème, de personnalité juridique et agissant implicitemente au nom de la communauté internationale et, de l'autre coté, Sa Majestaté Britannique, agissant pour le Gouvernenment de l'Afrique du Sud.

Les actes signés par la Société des Nations, tout en engageant les Estats-membres, ne pouvaient et ne peuvent pas être entendus et appliqués comme s'il s'agissait de 'Traités individuels ajustés entre ces Etats.

Le régime du Mandat est, done, une institution internationale, qui a créé un statut international définitif poưr le territoire du Sud-Ouest Africain, en imposant des obligations au Mandataire envers la communauté internationale, et non envers les anciens Etats-membres de la Société des Nations, individuellement considérés. Le droit de ces Etats au recours judiciaire, n'affecte du tout la valeu: de notre conclusion, car son emploi presuppose une violation spécifique, particulière, du Mandat et n'a rien de commun avec les facultés générales de surveillance appartenantes à la Société des Nations. Le mécanisme administratif du contrôle n'était pas exercé par la Société des Nations au nom individuel des Etats-membres; il l'était au nom de la Société même, en bénéfice et au profit de l'Humanité.

17. Nous n'ignorons pas que les Nations Unies ne se sont pas substituées automatiquement aux droits et aux devoirs de la Société des Nations; et nous savons même qu'aucun acte formel existe de transfert de ces droits ct de ces devoirs.

Mais une liaison a établie, quand même, au sujet des Mandats, entre la Société des Nations et les Nations Unies 
et cette liaison résulte de la Résolution de la Société, prise le 18 avril 1946, conçue en ces termes textuels:--

"Rappellant que l'art. 22 du Pacte applique à
certains territoires le principe que le bien-être
et le développement des peuples non encore ca-
pables de se diriger eux-mêmes dans les condi-
tions particulièrement difficiles du monde mo-
derne, forment une mission sacrée de civilisation:

(3) Reconnaît que la dissolution de la Société des Nations mettra fin à ses fonctions en ce qui concerne les territoires sous mandat, mais note que les principes correspondants à ceux que déclare l'art. $22 \mathrm{du}$ Pacte sont incorporés dans les chapitres XI, XII et XIII de la Charle des Nations Unies; (4) Note que les Membres de la Société, administrant actuellement des territoires sous Mandat, ont exprimé leur intention de continuer à l'administrer en vue $d u$ bien-être et développement des peuples interéssés, conformément aux obligations contenues dans les dixers Mandats, jusqu'à ce que des nouveaux arrangements soient pris entre les Nations Unies et les diverses Puissances Mandataires.

La Société des Nations, donc, lors de sa dissolution, par le vote de ses Membres parmi lesquels se trouvaient les Etats Mandataires, a reconnu et déclaré:

a) la cessation de ses fonctions, mais pas la cessation des Mandats;

b) la continuité des principes du Mandat dans la Charte des Nations Unies, c'est-à-dire, le caractère international de ces principes; 
c) l'acquiescence des Etats-Mandataires au maintien de ses obligations et l'autorité des Nations Unies pour la célébration de nouveaux arrangements, c'est-à-dire, la reconnaissance des Nations Unies comme le nouvel organe de la communauté internationale, devant lequel devraient être accomplies toutes les obligations résultantes du régime du Mandat.

Nous voici, donc, arrivés au moment de la création de la nouvelle situation juridique, à la naissance du successeur de la Société des Nations, muni des pouvoirs qui comprennent la possibilité légale de réaliser des nouveaux arrangements et de réclamer jusqu'à la célébration d'autres accords, sans solution de continuité, l'accomplissement des obligations des Etats Mandataires.

18. L'Union Sud-Africaine elle-même a reconnu et accepté cette conclusion, dans ses déclarations adressées à l'Assemblée Générale de la Société des Nations le 9 avril 1949, aussi bien que dans ses déclarations adréssées au Secrétaire Général le 5 novembre 1946.

À ce propos, la Cour Internationale de Justice rappelle aussi que, par letíre du 23 juillet 1947, adressée au Secrétaire Général des Nations Unies, la Légation de l'Afrique du Sud visa une Résolution du Parlement de ce pays qui déclarait:

"le gouvernement doit continuer à soumettre des rapports à l'Organisation des Nations Unies comme il l'avait fait jusqu'ici, en vertu du Mandat".

19. La valeur de toutes ces déclarations est plus forte encore que la valeur d'une simples interprétation donnée par la partie intéressée elle même, en tant qu'elles ont la signification et la force juridique d'une ratification. 
C'est un principe général, universel, de droit, celuı selon lequel l'acceptation des obligations par la partie intéressée corrige et reválide tous les défauts des actes juridiques qu’à ce obligations ont donné naissance.

Contre les effets de la ratification (entendue, non au sens formel et spécifique qui se rapporte à la mise en exécution de engagements, mais en un sens de principe général de droit), contre les effets de la ratification, une seule exception peut être opposée, fondée en défaut de compétence. Mais cette hypothèse, dans l'espèce que nous examinons, est à exclure, attendu qu'on ne peut pas mettro en doute les pouvoirs des signataires des déclarations mentionnées et moins encore les pouvoirs du parlement sud-africain.

Or, cette ratification a créé une situation juridique définitive, a rétabli, si l'on préfère dire, ces obligations et, en conséquence, aucun autre acte unilatéral de la partie engagée ne pourrait, plus tard ni jamais, résilier ces mêmes obligations, ni les altérer, non plus.

20. D'ailleurs, la nature définitive, irrévocable, de l'institution internationale de tutelle, comprise comme un ensemble des droits de la population du Sud-Ouest Africain et des droits et devoirs de la communauté internationale, aujourd'hui représentée par les Nations Unies, et, aussi, des droits et devoirs de l'Union Sud-Africaine, ne résulte seulement de la ratification mentionnée.

Aux droits des Nations Unies et de l'Etat Administrafeur correspondent les droits de la population du territoire, qui a reçu, ainsi, un statut international; et ces droits, une fois acquis, ne sont plus révocables. L'homme serait le plus malheureux des êtres, disait un vieil et savant juriste français, s'il ne pouvait être sûr, au moins, pour son passé. Et telle est la raison, profondément humaine, des garanties données, par les lois des pcuples 
libres, aux droits déjà acquis selon le droit en vigueur. au moment de l'acquisition.

Sir Arnold McNair, lui-même, nous rapporte à uñ décision de Marshall en ce sens:

"un droit, une fois conféré, n'exige pas, pour sa conservation, l'existence continue du pouvoir par lequel il a étẻ acquis. Si un traitẻ oui: toute autre loi a rempli sa fonction en accordant un droit, l'expiration du traité ou de la loi ne peut éteindre ce droit"

La dissolution, donc, de la Société des Nations, n'affecte en rien la continuité du règime de Mandat international, auquel est soumis le Sud-Ouest Africain. E ce régime comprend le contrôle administratif, sans lequel le régime lui-même serait affecté en sa substance.

21. Mais, quel est, donc, le contenu exact de ce Mandat? Est ce que ce mot désigne l'acte de délégation de pouvoirs, tout court, ou, plutôt, on doit le comprendro comme désignant, par entier, tout le régime international, auquel ce Mandat se rattache? - C'est la seconde signification que nous adoptons.

Les obligations résultantes du Mandat comprennent conséquemment, toutes les obligations:-

a) déclarées dans l'art. 22 du Pacte de la Société des. Nations;

b) déclarées dans l'acte du Mandat propremente dit;.

c) déclarées dans les résolutions et recommandations. des organes compétents de la Société des Nations, tels le Conseil, la Commission Permanente el l'Assemblée Générale; 
d) déclarées dans l'acceptation et dans les ratifica tions faites par l'Union Sud-Africaine, en ce qui concerne ses rapports d'Etat Mandataire avec les. Nations Unies.

Et c'est ainsi que parmi ces obligations figurent:

a) l'envoi des Rapports annuels aux Nations Unies. (ex-vi l'art. 10 de la Charte et les Résolutions dé l'Assemblée Générale 141-II, 227-III et 337-IV);

b) le renvoi à l'Assemblée Générale des Nations. Unies, avec les renseignements nécessaires, des pétitions des communatés ou des éléments de la population du territoire du Soud-Ouest Africain, ex-vi la décision du 31 janvier 1923 du Conseil de la Société des Nations et de l'art. 80 de la Charte des Nations Unies.

22. D'autres considérations suggère, encore, l'Avis de la Cour Internationale de Justice, telle la suivante:quelle est, en vue de la Charte des Nations Unies, la durée des obligations actuelles de l'Etat-Mandataire, c'est-à-dire, de l'Union de l'Afrique du Sud? Ces obligations continueront en viguer jusqu'au moment où l'Union aura conclu, avec les Nations Unies, un Accord de Tutelle qui contiendra, selon les chapitres XI, XII et XIII de la Charte, les droits et les devoirs de l'Etat-Administrateur.

23. La Cour a aussi déclaré qu'en vertu des arts. 75 et 77 de la Charte des Nations Unies, les Accords de Tutelle sont facultatifs, c'est-à-dire, pas obligatoires au sens légal. L'Afrique du Sud n'est pas liée par aucune: obligation légale (notez bien: - obligation légale) de conclure un accord de ce genre, et, en rapport à l'art. 81: de Ia Charte, il n'existe, non plus aucun moyen pour obliger l'Union à entamer des négociations pour cet Accord. 
24. S'il n'existe aucune obligation légale de soumettre le Sud-Ouest Africain au régime de tutelle, une obligation morale, de conscience, de confiance, toutefois existe pour que ces négociations soient entreprises sans retard. Au fait, le paragraphe 2 ème. de l'art. 2 de la Charte, dispose que l'Organisation des Nations Unies et ses Membres, dans la poursuite des buts énoncés à l'art. ler doivent agir conformément à certains principes, parmi lesquels s'inscrit celui-ci: - "les Membres de l'Organisation, afin d'assurer à tous la jouissance des droits et avantages résultants de leur qualité de Membre, doivent remplir de bonne foi les obligations qu'ils ont assumés aux termes de la présente Charte". Et l'art. 80, après avoir statué, dans son paragraphe ler. le maintien du statu quo antérieur à la célébration des Accords de Tutelle, ajoute, dans le paragraphe suivant: - "le paragraphe ler. du présent article ne doit pas être interpreté comme motivant un ajournement de la négociation et de la conclusion d'accords destinés à placer sous le régime de tutelle des territoires sous mandat, ou d'autres territoires, ainsi qu'il est prévu d̀ l'art. 7'7".

Nous ne nous rallions pas au point de vue de KELSEN, selon lequel ces mots "en bonne foi" sont superflus, parce qu'il est impossible de "remplir" une obligation en mauvaise foi. Nous ne nous rallions pas $\dot{x}$ ce point de vue, d'abord parce que toute interprétation doit donner un sens à tous les mots qui forment le texte de la loi; ensuite, parce que le paragraphe 2ème. de l'art. 2 , mis en rapport avec le paragraphe 2ème. de l'art. 80, autorise la conclusion suivante: - dans le système de la Charte, à la paire des obligations légales, d'autres obligations existent, qui relèvent de la bonne foi, de la morale, de la confiance, bref, de cette "equity" si feconde qui forme tout un droit dans le système juridique anglo-américain, auquel on peut, sans erreur, filier la Charte des Nations Unies. 
L'éminent juriste Sir Arnold McNair s'est bien rapporté au droit-équité quand il a cherché de rapprociner l'institution de la tutelle au "trust" et c'est à lui que nous empruntons ces trois principes dominants dans les affaires fiduciaires de ce genre, que ce droit aussi discipline:

“a) le contrôle des biens par le "trustee", tuteur ou curateur, est limité d'une manière ou d'une autre; le "trustee" n'est pas dans la situation du possesseur normal complet, qui "peut faire ce qu'il veut de sa propriété”, parce qu'il lui est interdit d'administrer les biens pour son avantage personnel;

“b) une sorte d'obligation juridique, une obligation de confiance et de conscience est imposée au "trustee", tuteur ou curateur, d'exercer le "trust" ou la mission qui luii est confiée dans l'intérét de quelque autre personne óu pour quelque fin d'intérét public";

“c) toute tentative de la part d'une de ces personnes en vue d'absorber dans son propre patrimoine les biens lui sont confiés serait illégale et prévenue par la loi".

C'est aussi "une sorte d'obligation juridique, une obligation de confiance et de conscience", inspirée par le principe de la bonne foi, que l'obligation de ne pas ajourner les négociations pour soumettre à un Accord de Tutelle le territoire, sous Mandat, du Sud-Ouest Africain.

Et l'Union Sud-Africaine, qui a une si solide tradition de scrupuleux respect pour ses engagements internationaux, qui a si loyalement participé du débat devant la Cour Internationale de Justice, ne tardera pas, certainement, a entamer les négociations nécessaires, en 
accomplissant, ainsi, cette obligation de confiance, de conscience, envers les Nations Unies.

\section{EN CONCLUTSION:}

La Délégation du Brésil, fidèle à son point de vue constamment manifesté, donnera son vote au sens: -

a) de l'adoption de l'Avts Facultatif de la Cour Internationale de Justice;

b) de la recommandation, à être adressée au Gouvernemente de l'Union de l'Afrique du Sud, pour entamer, sans retard, des négociations, en vue de la conclusion d'un Accord de Tutelle pour te territoire, actuellement sous Mandat, du Sud. -Ouest Africain;

c) du maintien, en plein vigueur, jusqu'à la conclusion de l'Accord, des obligations de l'Union de l'Afrique du Sud, résultantes du Mandat sur le territoire du Sud-Ouest Africain y incluse l'obligation d'envoyer à l'Assemblẻe Générale des $\mathrm{Na}$ tions Unies des Rapports Annuels et les pétitions des communautés ou des habitants de ce territoire, suivies des renseignements nécessaires.

L'autorité de la Cour Internationale de Justice est si notoire, M. le Président, que nous ne chercherons pas du tout de retenir vôtre attention sur l'importance de son Avis.

Le caractère facultatif de cet Avis ne diminue, en aucune façon, la valeur moral et juridique de l'opinion de cette Cour de Justice.

Et merci une fois encore pour votre bienveillante attention. 